\title{
Comparison of overall survival, local recurrence and distant metastasis of breast-conserving therapy with modified radical mastectomy in Iranian women with stage I and II breast cancer
}

\author{
Alireza Mozafari ${ }^{1}$, Fariba Binesh ${ }^{2,3}{ }^{*}$, Ali Akhavan ${ }^{4}$, Zeynab Eshaghie ${ }^{1}$, Mahyasadat Emami ${ }^{1}$, \\ Mahbubeh Haidari ${ }^{5}$ \\ 1, Medical Student, Student Research Committee, Shahid Sadoughi University of Medical Sciences, Yazd, Iran \\ 2 Department of Pathology, Shahid Sadoughi University of Medical Sciences, Yazd, Iran \\ 3 Hematology and Oncology Research Center, Shahid Sadoughi University of Medical Sciences and Health Services, Yazd, Iran \\ 4 Department of Radiotherapy, Isfahan University of Medical Sciences, Isfahan, Iran \\ 5 General Practitioner, Yazd, Iran \\ *Corresponding Author: Dr. Fariba Binesh, 1 Department of Pathology, Shahid Sadoughi University of Medical Sciences, Yazd, \\ Iran. 2 Hematology and Oncology Research Center, Shahid Sadoughi University of Medical Sciences and Health Services, Yazd, \\ Email: binesh44@yahoo.com; Tel: 983518113698
} Iran.

Received: April 02, 2020; Accepted: June 22, 2020

\section{Abstract}

Introduction: Breast cancer effects on younger patients and how it behaves more severely in Iran. This matter may influence the efficacy of breast conservation surgery (BCT) and radical mastectomy (MRM) as common treatments. The aim of this study was to compare the overall survival, local recurrence and distant metastasis of these two surgical methods in Iranian women with stage I and II of breast cancer.

Material and methods :This historical cohort study was performed on women with stage I\&ll of breast cancer who had undergone MRM and BCT and had referred to Shahid Ramezanzadeh Radiotherapy Center, Yazd, Iran, from 2005 to 2012.Patient's characteristics, tumor information, treatment modalities, local recurrence, distant metastasis and overall survival were collected. Data were analyzed by SPSS software using chisquare, t-test, log-rank and Kaplan-Meier tests. The study code of ethics is IR.SSU.MEDICINE.REC.1394.318.

Results: 506 patients were included in this study, 285 (56.3\%) in the MRM group and $221(43.7 \%)$ in the BCT group. The mean age of the patients was $47.69 \pm 10.62$ years. Most of the patients (408 patients or $80.6 \%$ ) were in stagell and the rest (98 patients or $19.4 \%$ ) were in stage lof the disease. Distant metastasis occurred in $23.94 \%$ (17 cases) of the patients in the BCT group and in $76.05 \%$ (54 cases) of the patients in the MRM group. The MRM group was indicative of more distant metastasis $(p=0.001)$. There was no significant difference between the two groups regarding local recurrence $(p=0.83)$. Overall survival in the BCT group was $105.43 \pm 2.06$ months and in the MRM group it was $96.26 \pm 2.16$ months, indicating a higher overall survival in the BCT group $(p=0.002)$.

Conclusion: The findings of the study showed that women with MRM had higher metastasis and lower overall survival. Also, local recurrence was not significantly different between the two groups. Considering the results, it is possible to recommend BCT as the treatment of choice for Iranian women.

Keywords: Breast carcinoma, recurrence, distant metastasis, mastectomy. 


\section{INTRODUCTION}

Breast cancer is the most common cancer in women and the most important cause of cancer death among them. Worldwide, physicians report about 1.7 million new cases of breast cancer annually, and almost every 18 seconds a new case is added [1]. Overall, one in 20 women develops breast cancer during her lifetime [2]. This figure increases to one in eight women in the United States [1]. In Iran too, it is the first cause of cancer in women and the fifth leading cause of cancer death [3]. Old age, BRCA1 and BRCA2 gene mutations, breast tissue density, age at the first menstruation, late menopause, use of estrogen and progestin hormones after menopause, inactivity and alcohol consumption are risk factors for breast cancer [1]. Although this cancer is more common in women who are menopause, it is more severe in premenopausal women, with a higher risk of recurrence, lymph node involvement and worse prognosis. [4]. As the disease progresses, metastasis to various organs such as bone, lung and brain occurs [5] and complications such as bone pain, hypercalcemia, and pathological fractures appear, which are ultimately fatal [1]. Unfortunately, in addition to the disease itself, the treatment also has many side effects; for example, chemotherapy and radiotherapy cause infertility and premature menopause and has unpleasant effects on the families [4].

MRM and BCT are common methods for early breast cancer treatment [6]. In MRM, the breast with ipsilateral axillary lymph nodes level I\&II are excised and the pector alis muscles remains [7]. In BCT lumpectomy along with the evaluation of the lymph nodes are performed and subsequently adjuvant radiotherapy is done [6, 7]. In BCT, a lumpectomy with at least $2 \mathrm{~mm}$ safe margin is effective in reducing the risk of recurrence of the disease [7]. Factors such as the size of the tumor, the importance of breast conservation for the patient, fear of radiotherapy, and the ease and completeness of treatment are effective in choosing the type of surgery [4, 7].The efficacy and superiority of the two mentioned methods of breast surgery in relation to each other have been compared in different studies [10 - 8]. In Iran due to the lack of screening programs for this disease, insufficient awareness and the lower age of breast cancer occurrence in comparison to western countries [11], causes Iranian women to experience a much more complicated illness with higher psychological consequences as well as higher recurrence [4]. This fundamental difference, which doubles the importance of the subject, led us to compare the efficacy and superiority of these two surgical methods in Yazd, Iran. The aim of this study was to compare the overall survival, local recurrence, and distant metastasis of BCT and MRM in women with breast cancer stage I and II.

\section{MATERIAL AND METHODS}

This is a historical cohort study with a 5-year follow up. Sampling was done through census. The statistical population consists of all females with stage I\&II of breast cancer who had undergone MRM and BCT and had referred to Shahid Ramazan Zadeh Radiotherapy Center, Yazd, Iran, from 2005 to 2012.Inclusion criteria were all females with stage I \&ll of breast cancer who had undergone MRM and BCT. Patients with lymph node involvement and metastasis at the time of diagnosis were excluded. Samples lacking information were not included in the study. Data were collected using the questionnaire based on the intended goals and it was done by the researcher using the information in the patients' records. Every patient had a file containing age, sex and tumor size, lymph node involvement, metastasis, stage of the disease, grade of the tumor, lymphovascular and perineural invasion, IHC results (Her2, ER, PR, Ki67), lymph node excision method, number of resected lymph nodes, contralateral breast involvement, type of breast surgery (BCT or MRM), adjuvant therapy (postoperative radiotherapy, postoperative chemotherapy, hormone therapy), local recurrence, distant metastasis and its location and the presence or absence of associated connective tissue diseases. Patients' follow-up was done on the phone. Overall survival was calculated from the day of diagnosis of breast cancer to death or last visit. Through the coordination with The Health Center of the Province, data on mortality demographics of the patients was obtained. The software of SPSS (version 17) was used to analyze the data. Chi-square tests was used for qualitative data analysis and T-test for quantitative data. Log-rank and KaplanMeier diagrams were used to determine and compare survival. Quantitative variables are reported as mean \pm SE and other variables are reported as percentages. P-value less than 0.05 was considered significant. In this study, 26 ethical codes were considered and we undertook not to disclose patient information. The study code of ethics is IR.SSU.MEDICINE.REC.1394.318

\section{RESULTS}

A total of 506 patients were included in the study, 285 (56.3\%) in the MRM group and 221 (43.7\%) in the BCT group. The minimum age of the patients was 22 years and their maximum age was 80 years with mean age $( \pm S E)$ of $47.69 \pm 10.62$ years. Most patients (408 patients or $80.6 \%$ ) were in stage II and the rest (98 patients or $19.4 \%$ ) were in stage 1 of the disease. Invasive ductal carcinoma comprised $78.3 \%$ of the tumors, invasive lobular carcinoma $8.9 \%$ and other type of tumors $12.8 \%$ (Table 1).

Distant metastasis occurred in $23.94 \%$ (17 cases) of the patients in the BCT group and $76.05 \%$ in the MRM (54 cases) group, this indicates a higher prevalence of distant metastasis in the MRM group $(p=0.001)$. In the age group under 40 years, distant metastasis occurred in $22.72 \%$ (5 cases) of the patients in the BCT group and $77.27 \%$ (17 cases) in the MRM group, this reveals that the risk of distant metastasis was significantly higher in patients under 40 years in the MRM group ( $p=0.002)$. Patients over 40 years in the BCT group also had distant metastases less than the MRM group $(p=0.01)$. There were no significant differences between the two groups in terms of local recurrence (Table 2 ).

Overall survival for all ages in the BCT group was 105.43 \pm 2.06 months and in the MRM it was $96.26 \pm 2.16$ months, this shows a higher overall survival in the BCT group $(p=0.002)$. In patients over 40 years, the overall survival in the MRM group was lower than that of the BCT $(105.83 \pm 2.33$ months in the BCT group versus $96.26 \pm 2.16$ months in the MRM group and $p=0.02$ ). Also in the age group under 40 years, overall survival was 103.9 \pm 3.57 months in the BCT group and $97.64 \pm 2.36$ months in the MRM group, this indicates that overall survival of the BCT group was significantly higher in this age range $(p=0.01)$. (Table 3 and Figures 1 to 3 .

\section{DISCUSSION}

The purpose of this study was to compare the rate of local recurrence, distant metastasis and overall survival of women with an early stage of breast cancer who had undergone two methods of surgery. Finally, we concluded that metastasis was greater in women treated by MRM and overall survival was lower. Also, local recurrence was not significantly different between the two groups. The best outcome for BCT in this study 
may be due to more patient referrals and stronger physician supervision or the effects of adjuvant radiotherapy after breast conservative surgery.

In the Hartman-Johnsen et al. cohort study of 13,015 Norwegian women who had undergone MRM and BCT in 2015 with a mean age of 59 years, the 5 -year survival rate in the BCT group was $97 \%$ and in the MRM group it was $88 \%$. They reported that cancer risk mortality ratio in patients treated by MRM was 1.64 compared to BCT. Also, overall survival in patients with BCT and MRM was $95 \%$ and $80 \%$, respectively. The results of this study indicate that the survival rate of BCT is similar or better than MRM in the early stages of the disease [8]. The present study confirms these results despite the young age of the patients.

In a clinical trial conducted by Litiere et al. in 2012, 868 patients with stage I\&II of breast cancer from 8 cancer centers in the United Kingdom, Belgium, the Netherlands and South Africa were enrolled in a 22.1-year median follow-up. Distant metastasis occurred in 175 (42\%) of the patients with MRM and $207(46 \%)$ of the patients with BCT. There was no significant difference in distant metastasis between the two groups. Overall survival was similar [9]. The risk of distant metastasis and overall survival of BCT was less in our study, suggesting that the duration of follow up is likely to be effective and that the shortand long-term outcomes may be different.

In a meta-analysis performed by Jatoi et al. in 2005, out of 6 clinical trials evaluated, 4 studies showed a significant decrease in locoregional recurrence in MRM compared to BCT. Only one clinical trial reported statistically significant effects of MRM on reducing patient mortality, but the odd ratio of mortality obtained from the meta-analysis was not statistically significant. This meta-analysis revealed similar effects of MRM and BCT on patient mortality even after prolonged follow-up, but ultimately the risk of recurrence after BCT was higher [10]. The difference between these results and our study may be due to older surgical techniques and surgeons' approach to the size of tumorfree margin excision during lumpectomy and its effect on regional recurrence. On the other hand, regional recurrence has been shown to be an independent prognostic factor for survival in a study [12] and may be an explanation for the discrepancy between the two studies.

Overall, the differences in the studies appear to be due to differences in the duration of follow up, treatment techniques, socioeconomic conditions, patients' awareness of the disease, and the amount of their cooperation with the physicians. In Iran, the disease has lower incidence but also lower survival [3, 13], so it is necessary to encourage women for breast examination and mammography from the fifth decade of life to increase their role in reducing the burden of the disease [14]. It should be noted that this study had some drawbacks:1-The lack of matching and short-term follow up resulted in limitations. 2- Missing data reduced the accuracy of this study, however.

\section{CONCLUSION}

In conclusion our findings show that the BCT results were more satisfactory. BCT can be considered as the treatment of choice for Iranian women in different age groups.

Prospective studies with longer follow up and matched confounder factors are suggested. BCT with safe surgical margins, consistent radiotherapy and chemotherapy approaches will be more illustrative.

This article is the result of the dissertation of $\mathrm{Dr}$ Mahboubeh Heydari, medical student of Shahid Sadoughi University of Medical Sciences, with tracking code 3844.

Table 1: Basic information

\begin{tabular}{|c|c|c|c|}
\hline Variable & Status & Number & Percent \\
\hline \multirow{2}{*}{ Type of surgery } & MRM & 285 & 56.3 \\
\hline & BCT & 221 & 43.7 \\
\hline \multirow{2}{*}{ Age (years) } & $40>$ & 126 & 24.9 \\
\hline & $40<$ & 380 & 75.1 \\
\hline \multirow{3}{*}{ Tumor size $(\mathrm{cm})$} & $2>$ & 121 & 23.9 \\
\hline & $2-5$ & 352 & 69.6 \\
\hline & $5<$ & 33 & 6.5 \\
\hline Involvement of lymph & - & 171 & 33.8 \\
\hline nodes & + & 335 & 66.2 \\
\hline \multirow{2}{*}{ Tumor Stage } & Stage 1 & 98 & 19.4 \\
\hline & Stage 2 & 408 & 80.6 \\
\hline \multirow{3}{*}{ Type of tumor } & $\begin{array}{l}\text { Invasive ductal } \\
\text { carcinoma }\end{array}$ & 396 & 78.3 \\
\hline & $\begin{array}{l}\text { Invasive lobular } \\
\text { carcinoma }\end{array}$ & 45 & 8.9 \\
\hline & Other & 65 & 12.8 \\
\hline \multirow{4}{*}{ Tumor grade } & 1 & 37 & 7.3 \\
\hline & 2 & 229 & 45.3 \\
\hline & 3 & 114 & 22.5 \\
\hline & Not reported & 126 & 24.9 \\
\hline Lymphatic-vascular and & Not involved & 362 & 71.5 \\
\hline
\end{tabular}




\begin{tabular}{|c|c|c|c|}
\hline \multirow[t]{4}{*}{ nerve invasion } & $\begin{array}{c}\text { Lymphatic-vascular } \\
\text { invasion }\end{array}$ & 65 & 12.8 \\
\hline & nerve invasion & 49 & 9.7 \\
\hline & Both & 30 & 5.9 \\
\hline & Less than $15 \%$ positive & 103 & 20.3 \\
\hline \multirow[t]{3}{*}{ Ki67 marker } & More than $15 \%$ positive & 96 & 19 \\
\hline & Not reported & 307 & 60.7 \\
\hline & - & 250 & 49.4 \\
\hline \multirow[t]{2}{*}{ Her2 marker } & + & 111 & 21.9 \\
\hline & Not reported & 145 & 28.7 \\
\hline Her2 marker verification & $3+$ in $\mathrm{IHC}$ & 94 & 84.6 \\
\hline \multirow[t]{2}{*}{ method } & $\mathrm{FISH}$ & 17 & 15.4 \\
\hline & - & 148 & 29.2 \\
\hline \multirow[t]{3}{*}{ ER } & + & 253 & 50 \\
\hline & Not reported & 105 & 20.8 \\
\hline & - & 170 & 33.6 \\
\hline \multirow[t]{2}{*}{ PR } & + & 231 & 45.7 \\
\hline & Not reported & 105 & 20.7 \\
\hline Type of lymph node & Sentinel & 2 & 0.4 \\
\hline resection & LND & 504 & 99.6 \\
\hline \multirow{3}{*}{$\begin{array}{l}\text { Number of resected } \\
\text { lymph nodes }\end{array}$} & $10>$ & 227 & 44.8 \\
\hline & $10<$ & 100 & 19.8 \\
\hline & Not reported & 179 & 35.4 \\
\hline The opposite breast & - & 501 & 99 \\
\hline involvement & + & 5 & 1 \\
\hline \multirow{3}{*}{ Radiotherapy } & - & 3 & 0.6 \\
\hline & + & 503 & 99.4 \\
\hline & - & 1 & 0.2 \\
\hline \multirow[t]{2}{*}{ Chemotherapy } & + & 465 & 91.9 \\
\hline & Not reported & 40 & 7.9 \\
\hline \multirow{5}{*}{ Hormone therapy } & + & 195 & 38.5 \\
\hline & Not reported & 311 & 61.5 \\
\hline & Brain & 20 & 4 \\
\hline & Bone & 33 & 6.5 \\
\hline & Liver & 8 & 1.6 \\
\hline \multirow{4}{*}{$\begin{array}{c}\text { Distant metastasis } \\
\text { location }\end{array}$} & Lung & 6 & 1.2 \\
\hline & The opposite breast & 2 & 0.4 \\
\hline & Other & 3 & 0.6 \\
\hline & Not reported & 434 & 85.7 \\
\hline \multirow{2}{*}{ Life status } & Alive & 412 & 81.4 \\
\hline & Dead & 94 & 18.6 \\
\hline
\end{tabular}

ER: Estrogen receptor

PR: Progesterone receptor

LND: Iymph node dissection 
Table 2: Determination and comparison of local recurrence and distant metastasis in both BCT and MRM methods

\begin{tabular}{ccccc}
\hline Variable & Age Interval & BCT & MRM & P Value \\
\hline \multirow{3}{*}{ Local recurrence } & $40>$ & $55.55 \%(5)$ & $44.44 \%(4)$ & 0.88 \\
& $40<$ & $40.00 \%(6)$ & $60.00 \%(9)$ & 0.97 \\
& All ages & $45.83 \%(11)$ & $54.16 \%(13)$ & 0.83 \\
\multirow{2}{*}{ Distant metastasis } & $40>$ & $22.72 \%(5)$ & $77.27 \%(17)$ & 0.002 \\
& $40<$ & $24.48 \%(12)$ & $75.51 \%(37)$ & 0.01 \\
& All ages & $23.94 \%(17)$ & $76.05 \%(54)$ & 0.001 \\
\hline
\end{tabular}

BCT: breast conserving therapy

MRM: modified radical mastectomy

Data were reported as percentage (number).

$P$ value was calculated by Chi-Square test and $p$ value less than 0.05 was considered significant

Table 3: Comparison of overall survival (months) in both BCT and MRM methods

\begin{tabular}{|c|c|c|c|}
\hline Age Interval & BCT & MRM & P Value \\
\hline $40>$ & $103.9 \pm 3.57$ & $89.72 \pm 5.07$ & 0.01 \\
\hline $40<$ & $105.83 \pm 2.33$ & $97.64 \pm 2.36$ & 0.02 \\
\hline All ages & $105.43 \pm 2.06$ & $96.26 \pm 2.16$ & 0.002 \\
\hline
\end{tabular}

BCT: breast conserving therapy

MRM: modified radical mastectomy

Overall survival was reported as SE \pm mean from 506 patients.

$P$ value was calculated by Log-rank test and $p$ value less than 0.05 was considered significant. 


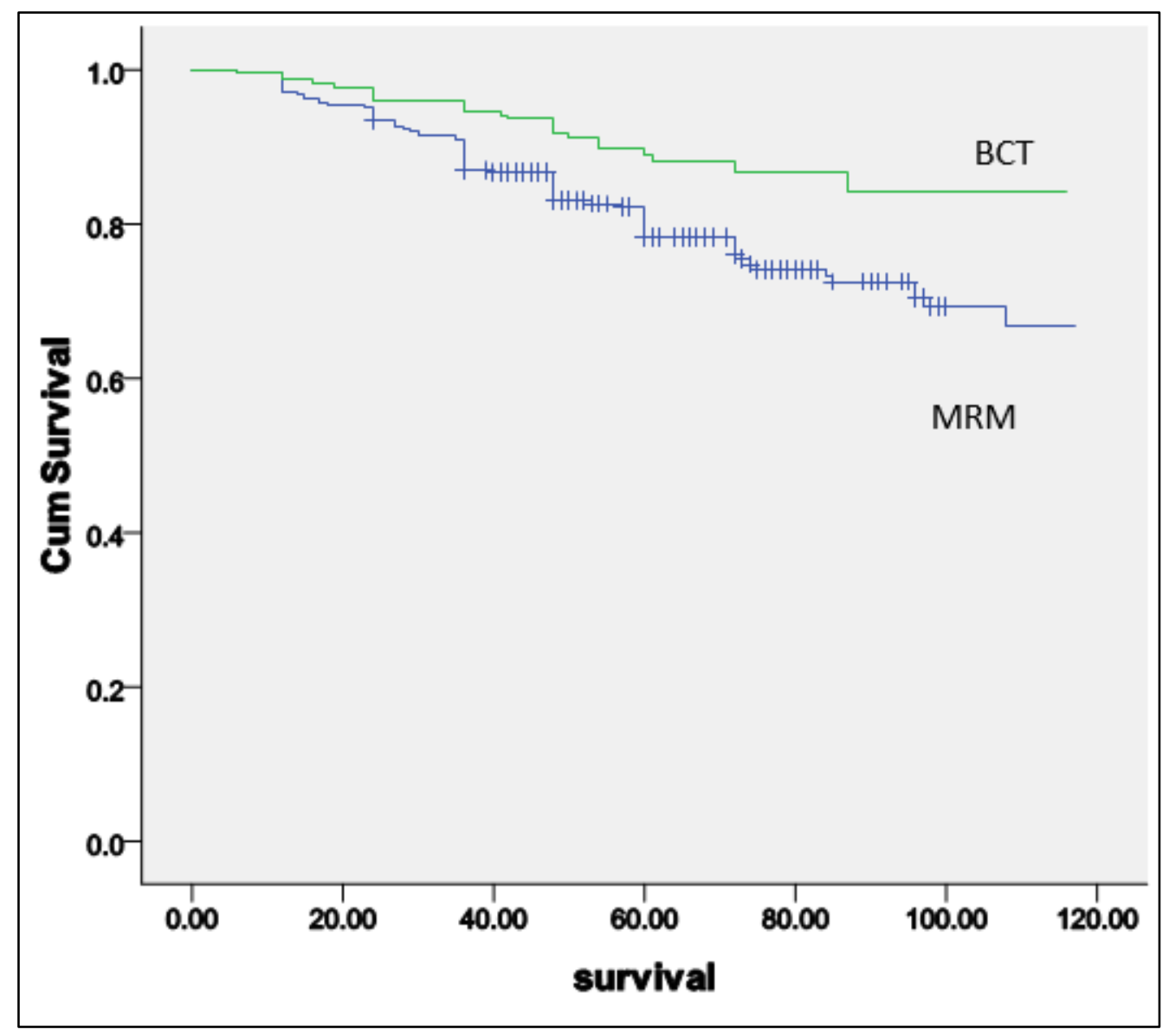

Fig. 1: Overall Survival Estimated by BCT and MRM in patients with breast carcinoma

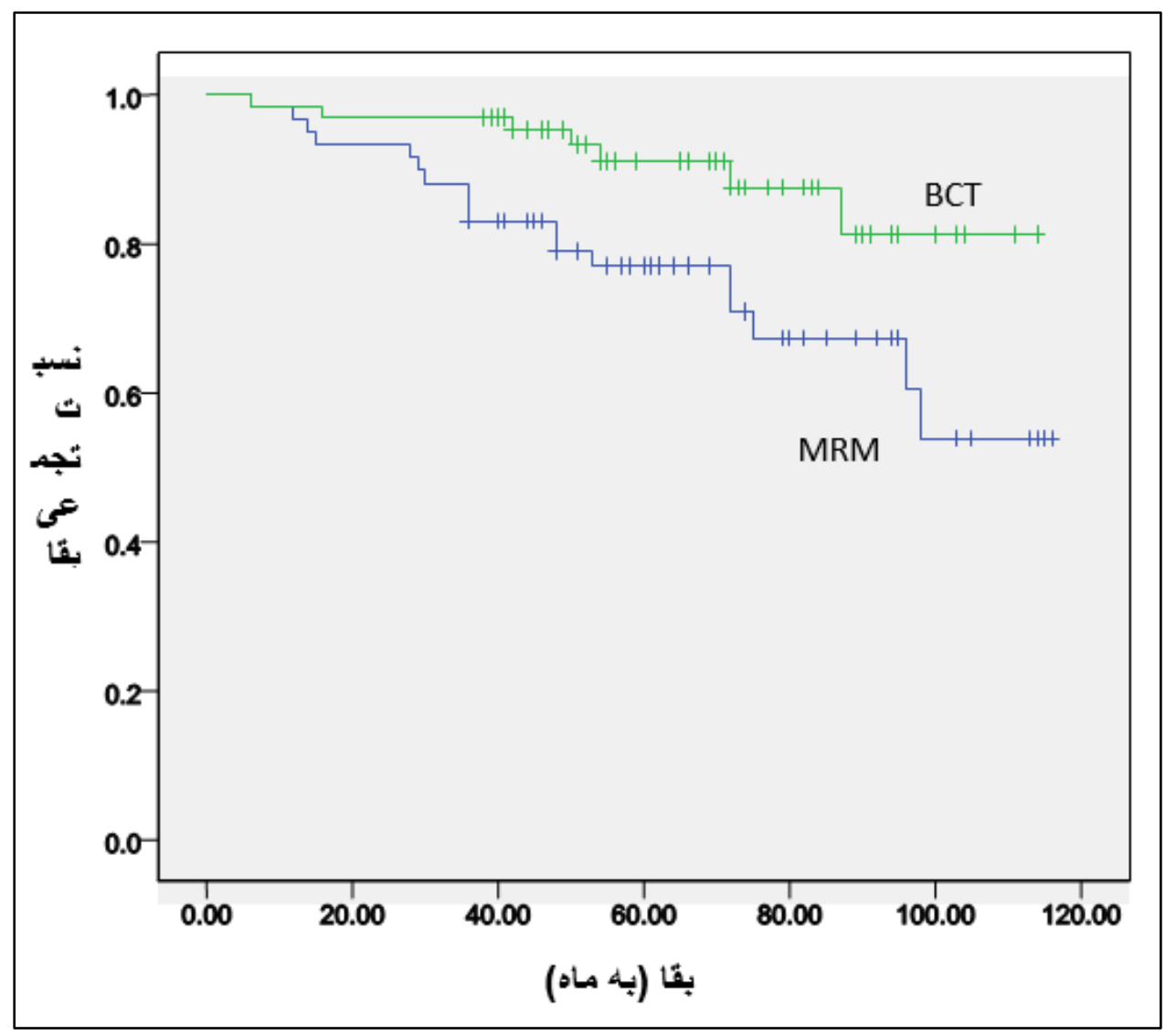


Fig 2: Overall survival Estimated by BCT and MRM in age group under 40 years

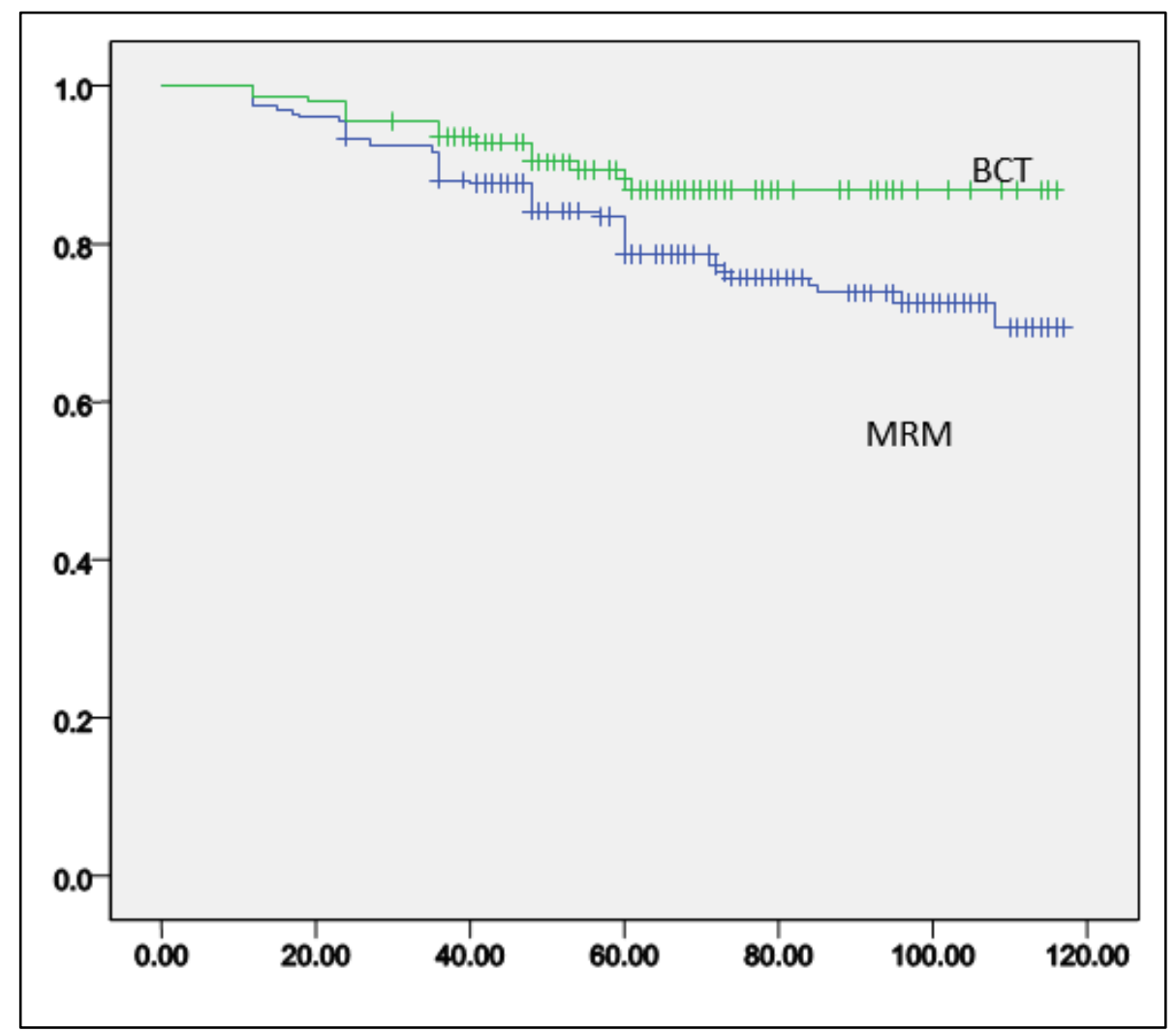

Fig 3: Overall survival Estimated by BCT and MRM in age group over 40 years

\section{Acknowledgements}

The authors of this article would like to thank the staff of Ramazanzadeh Radiotherapy Center, for their support and help.

Contributions: All the authors contributed in the preparation of this research and article.

\section{Conflict of Interest: None.}

\section{REFERENCES}

1. Winters S, Martin C, Murphy D, Shokar NK. Breast cancer epidemiology, prevention, and screening. Progress in molecular biology and translational science. 151: Elsevier; 2017, 1-32.

2. Global Burden of Disease Cancer C, Fitzmaurice C, Akinyemiju TF, Al Lami FH, Alam T, Alizadeh-Navaei R, et al. Global, Regional, and National Cancer Incidence, Mortality, Years of Life Lost, Years Lived With Disability, and Disability-Adjusted Life-Years for 29 Cancer Groups, 1990 to 2016: A Systematic Analysis for the Global Burden of Disease Study. JAMA oncology. 2018;4(11):1553-68.

3. Farhood B, Geraily G, Alizadeh A. Incidence and Mortality of Various Cancers in Iran and Compare to Other Countries: A Review Article. Iranian journal of public health. 2018;47(3):309-16.

4. Recio-Saucedo A, Gerty S, Foster C, Eccles D, Cutress RI. Information requirements of young women with breast cancer treated with mastectomy or breast conserving surgery: A systematic review. Breast (Edinburgh, Scotland). 2016;25:1-13.

5. Kozlowski J, Kozlowska A, Kocki J. Breast cancer metastasis - insight into selected molecular mechanisms of the phenomenon. Postepy higieny i medycyny doswiadczalnej (Online). 2015;69:447-51.

6. Bao X, Sun K, Tian X, Yin Q, Jin M, Yu N, et al. Present and changing trends in surgical modalities and neoadjuvant chemotherapy administration for female breast cancer in Beijing, China: A 10-year (2006-2015) retrospective hospitalization summary report-based study. Thoracic cancer. 2018;9(6):707-17.

7. Cil TD, McCready D. Modern Approaches to the Surgical Management of Malignant Breast Disease: The Role of Breast Conservation, Complete Mastectomy, Skin- and Nipple-Sparing Mastectomy. Clinics in plastic surgery. 2018;45(1):1-11.

8. Hartmann-Johnsen OJ, Karesen R, Schlichting E, Nygard JF. Survival is Better After Breast Conserving Therapy than Mastectomy for Early Stage Breast Cancer: A RegistryBased Follow-up Study of Norwegian Women Primary Operated Between 1998 and 2008. Annals of surgical oncology. 2015;22(12):3836-45.

9. Litiere S, Werutsky G, Fentiman IS, Rutgers E, Christiaens MR, Van Limbergen E, et al. Breast conserving therapy versus mastectomy for stage I-II breast cancer: 20 year follow-up of the EORTC 10801 phase 3 randomised trial. Lancet Oncol. 2012;13(4):412-9.

10. Jatoi I, Proschan MA. Randomized trials of breastconserving therapy versus mastectomy for primary breast cancer: a pooled analysis of updated results. Am J Clin Oncol. 2005;28(3):289-94.

11. Sharifian A, Pourhoseingholi MA, Emadedin M, Rostami 
Nejad M, Ashtari S, Hajizadeh N, et al. Burden of Breast Cancer in Iranian Women is Increasing. Asian Pacific journal of cancer prevention : APJCP. 2015;16(12):504952.

12. Xie Z, Wang X, Lin H, Wei W, Liu P, Xiao X, et al. Breastconserving therapy: a viable option for young women with early breast cancer--evidence from a prospective study. Annals of surgical oncology. 2014;21(7):2188-96.

13. Rezaianzadeh A, Jalali M, Maghsoudi A, Mokhtari AM, Azgomi SH, Dehghani SL. The overall 5-year survival rate of breast cancer among iranian women: A systematic review and meta-analysis of published studies. Breast disease. 2017;37(2):63-8.

14. da Costa Vieira RA, Biller G, Uemura G, Ruiz CA, Curado MP. Breast cancer screening in developing countries. Clinics (Sao Paulo, Brazil). 2017;72(4):244-53. 